\title{
Expression of human ARGONAUTE 2 inhibits endogenous microRNA activity in Arabidopsis
}

\author{
Ira Deveson, Junyan Li and Anthony A. Millar* \\ Plant Sciences Division, Research School of Biology, Australian National University, Canberra, ACT, Australia
}

\section{Edited by:}

Jean-Philippe Vielle-Calzada,

CINVESTAV, Mexico

\section{Reviewed by:}

Javier Palatnik, Instituto de Biologia

Molecular y Celular de Rosario,

Argentina

Mario A. Arteaga-Vazquez,

Universidad Veracruzana, Mexico

*Correspondence:

Anthony A. Millar, Plant Sciences Division, Research School of

Biology, Australian National

University, Building 134, Linnaeus

Way, Canberra 0200, ACT, Australia.

e-mail: tony.millar@anu.edu.au
Plant and animal microRNA (miRNA) pathways share many analogous components, the ARGONAUTE (AGO) proteins being foremost among them. We sought to ascertain the degree of functional conservation shared by Homo sapiens ARGONAUTE 2 (HsAGO2) and Arabidopsis thaliana ARGONAUTE 1 (AtAGO1), which are the predominant AGO family members involved with miRNA activity in their respective species. Transgenic Arabidopsis plants expressing HsAGO2 were indistinguishable from counterparts over-expressing AtAGO1, each group exhibiting the morphological and molecular hallmarks of miRNA-pathway loss-of-function alleles. However, unlike AtAGO1, HsAGO2 was unable to rescue the ago1-27 allele. We conclude that, despite the evolutionary gulf between them, HsAGO2 is likely capable of interacting with some component/s of the Arabidopsis miRNA pathway, thereby perturbing its operation, although differences have arisen such that HsAGO2 alone is insufficient to confer efficient silencing of miRNA targets in planta.

Keywords: HsAGO2, AtAGO1, ago 1-27, microRNA, Arabidopsis

\section{INTRODUCTION}

MicroRNAs (miRNAs) are endogenous small RNAs (sRNAs) that direct the sequence-specific silencing of mRNA transcripts, providing a critical layer of gene regulation in both plants and animals (Pasquinelli, 2012). Much of the molecular machinery that oversees miRNA biogenesis and activity is shared between these kingdoms and, hence, must have arisen prior to their divergence, though it is unclear whether a basal miRNA pathway was already in operation at this time (Axtell et al., 2011). From this shared origin, the plant and animal miRNA pathways have followed divergent evolutionary courses and there now exists characteristic distinctions in the manner of their operation. Briefly, plant miRNAs generally silence only a small handful of targets, requiring near-perfect complementarity for their recognition, and do so via a combination of transcript cleavage and a non-cleavage mechanism/s (Brodersen and Voinnet, 2009). In contrast, animal miRNAs regulate target transcripts to which they are only partially complementary, generally recognizing many targets and silencing these via a non-cleavage mechanism/s (Axtell et al., 2011; Huntzinger and Izaurralde, 2011). The question of how the apparently similar set of components in the miRNA pathways of plants and animals has been modified so as to generate these functional differences is keenly relevant to our understanding of eukaryotic gene regulation.

The best example of shared componentry between the systems, an ARGONAUTE (AGO) protein is required, without known exception, for miRNA-mediated gene silencing (Mallory and Vaucheret, 2010). Considered the core component of the miRNA induced silencing complex (miRISC), AGO's involvement in miRNA-mediated silencing appears threefold. First, it facilitates the interaction of a miRNA with its intended targets (Nakanishi et al., 2012; Schirle and Macrae, 2012). Second, when sufficient complementarity exists, it provides the catalytic activity for cleavage of the target mRNA (Liu et al., 2004; Meister et al., 2004; Baumberger and Baulcombe, 2005; Rivas et al., 2005). Finally, it orchestrates the silencing of un-cleaved targets by recruiting the factors responsible for their translational repression and accelerated degradation (Huntzinger and Izaurralde, 2011).

Plants and animals each exhibit a diversity of AGO proteins. There are, for example, 10 members in Arabidopsis, which are distinguished by the class of sRNAs that they associate with and by their patterns of expression (Vaucheret, 2008; Mallory and Vaucheret, 2010). Of these, Arabidopsis thaliana AGO1 (AtAGO1) has the most prominent involvement in miRNA-mediated gene silencing. Emphasizing the importance of its role, AtAGO1 homeostasis must be delicately maintained by a feedback mechanism in which miR168 directs the AtAGO1-dependent silencing of AtAGO1 expression. When this circuit is disturbed via the mutation of AtAGO1's miR168 target site, the over-accumulation of AtAGO1 protein leads-somewhat counter intuitively-to a general perturbation of miRNA-mediated regulation in the plant, manifesting in pleiotropic defects that resemble those apparent in a range of miRNA-pathway loss-of-function alleles (Morel et al., 2002; Vaucheret et al., 2004; Vazquez et al., 2004).

Of four mammalian AGOs, Homo sapiens AGO2 (HsAGO2) has the best-characterized role in the miRNA pathway. Although HsAGO2 and AtAGO1 share only $43 \%$ amino acid identity (Poulsen et al., 2013), they appear similar in function. Both preferentially associate with sRNAs bearing a $5^{\prime} \mathrm{U}$ (Mi et al., 2008; Frank et al., 2010) and may catalyze the cleavage of complementary mRNA transcripts in vitro without the participation of any other component (Liu et al., 2004; Meister et al., 2004; Baumberger and Baulcombe, 2005; Rivas et al., 2005). Moreover, they interact and operate in conjunction with proteins of analogous structure and function. For example, HsAGO2 facilitates 
the non-cleavage silencing of targets (Pillai et al., 2004) in a process dependent on the GW182 family proteins (Takimoto et al., 2009; Zipprich et al., 2009), while AtAGO1 (Brodersen et al., 2008; Lanet et al., 2009) and an Arabidopsis GW protein SUO (Yang et al., 2012) each have a demonstrated involvement in miRNA-mediated translational repression. The plant de-capping factor VARCIOSE (VCS) (Brodersen et al., 2008) and its animal ortholog Ge-1 (Eulalio et al., 2007) have also been implicated in translational repression. Further to this, the loading of miRNAs into AtAGO1 and HsAGO2 is supported by chaperones that are conserved between the two kingdoms (Smith et al., 2009; Iki et al., 2010, 2012; Iwasaki et al., 2010; Earley and Poethig, 2011).

The array of similarities just outlined indicates that, despite the evolutionary distance between them, AtAGO1 and HsAGO2 share a remarkable degree of functional conservation. Indeed, in their recent report, Poulsen et al. (2013) integrated information from mutational studies of AtAGO1 with structural studies of HsAGO2 to make general inferences about the mechanics of AGO activity, the underlying assumption being that these two proteins are highly similar in form and function. By expressing HsAGO2 in Arabidopsis, we sought to determine whether they might be so similar that HsAGO2 could function as a component of the plant miRNA pathway or whether distinctions that have accumulated during their parallel evolution would become apparent when expressed in an identical cellular context, perhaps providing insight into the characteristic operational differences of the plant and animal miRNA pathways.

\section{MATERIALS AND METHODS \\ PLANT MATERIALS AND GROWTH CONDITIONS}

Seeds were surface sterilized by exposure (3-6h) to the chlorine gas generated by mixing $100 \mathrm{~mL}$ of sodium hypochlorite with $3 \mathrm{~mL}$ of concentrated hydrochloric acid in a sealed desiccator jar. Seeds were sown on soil (Debco Plugger mixed with Osmocote Extra Mini fertilizer at $3.5 \mathrm{~g} / \mathrm{L}$ ), stratified for $48 \mathrm{~h}$ at $4^{\circ} \mathrm{C}$ and grown under "long day" conditions ( $16 \mathrm{~h}$ light $/ 8 \mathrm{~h}$ dark, $\left.150 \mu \mathrm{mol} / \mathrm{m}^{2} / \mathrm{s}, 22^{\circ} \mathrm{C}\right)$.

\section{GENERATING 35S:HsAG02, 35S:AtAG01 AND 35S:4m-AG01 TRANSGENIC PLANTS}

HsAGO2 and AtAGO1 cDNAs were obtained from Sino Biological and the Arabidopsis Biological Resource Centre respectively. Via standard Gateway cloning procedures, each was placed into the pMDC32 destination vector, which contains a double $35 \mathrm{~S}$ promoter for constitutive expression (Curtis and Grossniklaus, 2003). For the 35S:4mAGO1 construct, four silent mutations introducing mismatches to the miR168 target site in AtAGO1, as per (Vaucheret et al., 2004), were generated via a site directed mutagenesis strategy based on that of (Liu and Naismith, 2008). Constructs were transformed into Agrobacterium tumefaciens and then transformed into Arabidopsis using the "floral dip" method (Clough and Bent, 1998).

\section{RNA EXTRACTION AND cDNA SYNTHESIS}

Total RNA was extracted from whole rosettes of plants at different growth stages, using TRIzol (Invitrogen) with the following modifications made to the manufactures protocol: (1) approximately $500 \mathrm{mg}$ of plant material was used with $1 \mathrm{~mL}$ of Trizol reagent for each extraction; (2) homogenization of tissues was achieved using a mortar and pestle; (3) the chloroform extraction step was repeated twice; (4) precipitation of RNA was carried out overnight at $-20^{\circ} \mathrm{C}$ to maximize the recovery of sRNAs. 30-50 $\mu \mathrm{g}$ of RNA from each sample was treated with RQ1 RNaseFree DNase (Promega) in separate $100 \mu \mathrm{L}$ reactions, according to the manufacturer's protocol, with the addition of RNaseOut Recombinant RNase Inhibitor (Invitrogen) ( $1 \mu \mathrm{L} / 10 \mu \mathrm{g}$ RNA). Treated RNA was then purified using Qiagen RNAeasy cleanup kit according to the manufacturer's protocol. cDNA synthesis was carried out using SuperScript III Reverse Transcriptase (Invitrogen) and an oligo dT primer according to manufacturer's protocol. For each sample, $250 \mathrm{ng}-5 \mu \mathrm{g}$ of purified RNA was used in separate $20 \mu \mathrm{L}$ reactions. These were subsequently diluted in $980 \mu \mathrm{L}$ nuclease free distilled water before qRT-PCR analysis.

\section{QUANTITATIVE REAL-TIME PCR (qRT-PCR)}

For qRT-PCR, $9.2 \mu \mathrm{L}$ of each cDNA sample was added to $10 \mu \mathrm{L}$ of SensiFAST SYBR No-ROX mix (Bioline) with $0.8 \mu \mathrm{L}$ of forward and reverse primers $(10 \mu \mathrm{mol}$ each). For the measurement of un-cleaved mRNA levels of miRNA target genes, qRT-PCR primers were designed so their amplicon would span the target site for their associated miRNAs, meaning that cleaved transcripts would not contribute to the measured abundance. qRT-PCR reactions were carried out on a Rotor-Gene 2000 real time PCR machine (Qiagen) in triplicate. The "housekeeper" CYCLOPHILIN (At2g29960) was used to normalize mRNA levels of each gene using the comparative quantitation program in the Rotor-Gene 6 software package provided by Qiagen and average values were calculated from triplicate measurements.

\section{qRT-PCR ASSAYS FOR MATURE miRNAs}

Customized Taqman sRNA assays (Applied Biosystem) were used to quantitate mature miRNAs and amiRNAs according to the manufacturer's protocol, apart from the following modifications: (1) for each RNA sample, the retro-transcription was multiplexed with looped-RT primers for the miRNA/amiRNA of interest and the small RNA sno101; (2) the cDNA synthesized $(15 \mu \mathrm{L})$ was diluted with $86.4 \mu \mathrm{L}$ nuclease free distilled water and $9 \mu \mathrm{L}$ of this cDNA solution was used in each qRT-PCR reaction, along with $10 \mu \mathrm{L}$ SensiFAST Probe NO-ROX mix (Bioline) and $1 \mu \mathrm{L}$ Taqman probe (Applied Biosystems). qRT-PCR reactions were carried out on a Corbett Rotor-Gene 2000 real time PCR machine (Corbett) in triplicate as above. The abundance of each mature miRNA/amiRNA was normalized to sno101 using the comparative quantitation analysis program from Rotor-Gene 6 software (Corbett) and average values were calculated from triplicate measurements.

\section{RESULTS EXPRESSION OF HsAG02 IN Arabidopsis RESULTS IN PLEIOTROPIC DEVELOPMENTAL DEFECTS}

A cDNA for HsAGO2 was placed under the control of a double $35 \mathrm{~S}$ promoter and the resulting binary vector (35S:HsAGO2) was 
transformed into wild type Arabidopsis. Roughly half (17/35) of all 35S:HsAGO2 primary transformants displayed morphological abnormalities of varying severity. At its mildest, the 35S:HsAGO2 phenotype was characterized by increased leaf-serration relative to wild type. Reduced rosette size, accelerated senescence and flat, broad leaves were additional features in more severely affected transformants and, in several, upward leaf-curl was also apparent (Figure 1A). Plants were grouped according to the perceived severity of their abnormalities and these assignments were well correlated with the abundance of $\mathrm{HsAGO} 2$ mRNA recorded in each set (Figure 1B). Hence, the expression of $\mathrm{HsAGO} 2$ is able to generate pleiotropic developmental defects in Arabidopsis.

\section{HsAG02 EXPRESSION AND AtAG01 OVER-EXPRESSION GENERATE INDISTINGUISHABLE MORPHOLOGICAL PHENOTYPES IN Arabidopsis}

The serration and upward leaf-curl seen in 35S:HsAGO2 transgenic plants are features observed in a number of previously described miRNA-pathway loss-of-function alleles (Morel et al., 2002; Vaucheret et al., 2004; Vazquez et al., 2004), hinting that endogenous miRNA activity might be perturbed by $\mathrm{HsAGO} 2$ expression. Since over-expression of endogenous AtAGO1, elicited by the mutation of its miR168 target site, leads to a miRNA-pathway loss-of-function effect (Vaucheret et al., 2004, 2006), the excessive expression of HsAGO2, unregulated by any homeostatic mechanism, might perturb miRNA activity in a similar fashion. To test this, we wished to directly compare 35S:HsAGO2 plants to transgenic plants over-expressing AtAGO1. To generate these, a cDNA for AtAGO1 was placed under a double $35 \mathrm{~S}$ promoter (35S:AtAGO1) and, in a separate construct (35S:4mAGO1), four silent mutations identical to those described by Vaucheret et al. (2004) were created, introducing four mismatches to AtAGO1's miR168 target site, which should render it resistant to miR168-mediated regulation. Both constructs were transformed into wild type plants and their primary transformants were grown alongside 35S:HsAGO2 plants. Populations of transformants for all three constructs were morphologically indistinguishable, each eliciting phenotypes characterized by serration and broad, flattened leaves (Figure 2A). These abnormal phenotypes were associated with elevated AtAGO1 mRNA levels (Figure 2B), ruling out co-suppression of the endogenous gene as an alternative explanation. The similarity of plants expressing $\mathrm{HsAGO} 2$ to plants over-expressing AtAGO1 is consistent with the possibility that $\mathrm{HsAGO} 2$ expression perturbs endogenous miRNA activity and suggests that the HsAGO2 protein behaves similarly to AtAGO1 in an over-expression context.

\section{HsAG02 PLANTS EXHIBIT MOLECULAR CHARACTERISTICS OF miRNA-PATHWAY LOSS-OF-FUNCTION ALLELES}

Seeking further evidence that $\mathrm{HsAGO} 2$ expression perturbs endogenous miRNA activity, the abundance of un-cleaved mRNA for each of five miRNA targets was measured in $\mathrm{HsAGO} 2$ transformants that displayed obvious or mild/no morphological abnormalities. The same analysis was performed for 35S:AtAGO1 and 35S:4mAGO1 transformants, with wild type plants and ago1-27, a partial
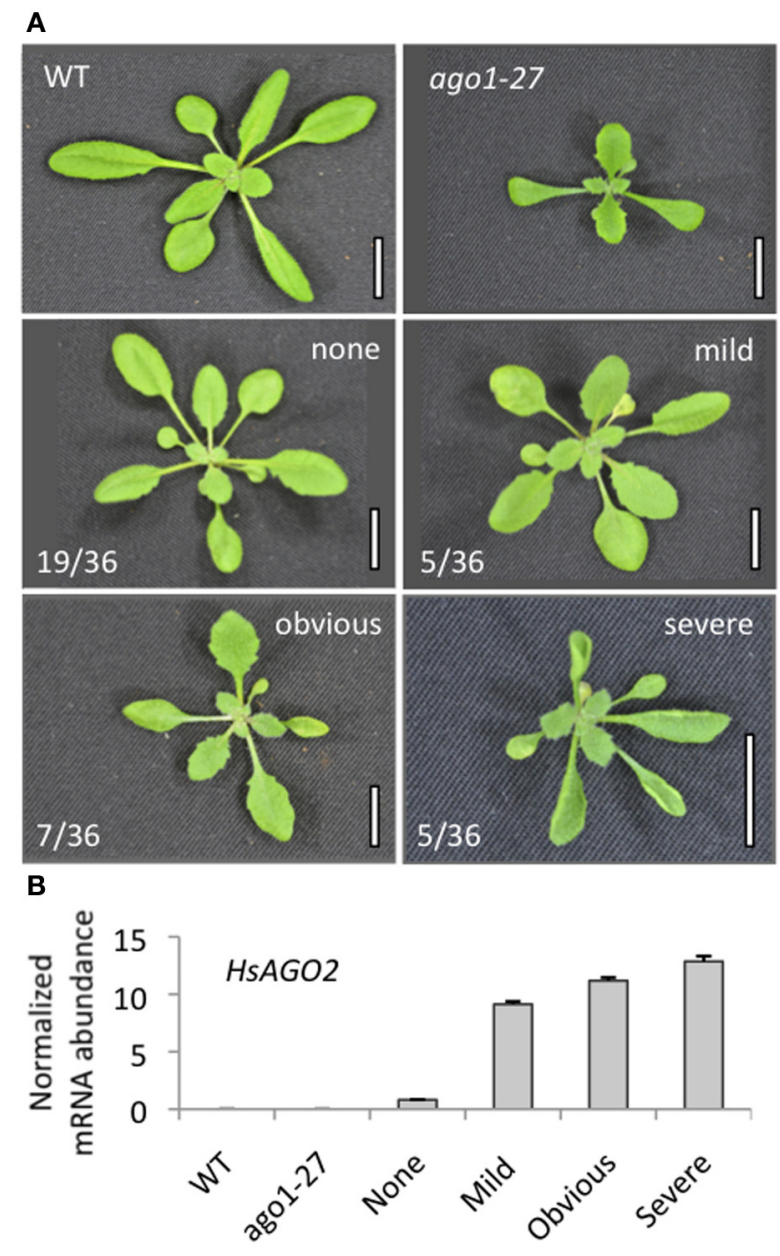

FIGURE 1 | Expression of HsAGO2 generates morphological defects in Arabidopsis. (A) 22-day old primary transformants for the 35S:HsAGO2 construct were categorized based on the apparent severity of their morphological phenotypes. Increased leaf serration distinguished "mild" phenotypes from wild type (WT), "obvious" phenotypes were characterized by broadened leaves, serration, accelerated senescence and some upward leaf-curl, while phenotypes considered "severe" were distinguished by strong upward leaf-curl in addition. Scale bars represent $10 \mathrm{~mm}$. (B) The abundances of $\mathrm{HsAGO} 2$ mRNA was measured in total RNA from sample pools composed of 4-8 transformants, 22-days old, from each morphological category. Wild type (WT) and ago1-27 plants, grown in parallel, were included as controls. All measurements are normalized to CYCLOPHILIN mRNA. Data is averaged from three technical cDNA replicates, each of which comprised triplicate measurements, and error bars depict standard error of the mean.

loss-of-function allele of AtAGO1 (Morel et al., 2002), serving as controls.

The five miRNA targets examined, AtAGO1 (miR168), DCL1 (miR162), PHABULOSA (PHB) (miR165/166), MYB33 (miR159), and CUP-SHAPED COTYLEDONS 2 (CUC2) (miR164), all showed elevated un-cleaved mRNA abundances in ago1-27 plants relative to wild type (Figure 2B), consistent with what has previously been reported (Morel et al., 2002). HsAGO2 plants displaying obvious aberrant phenotypes also exhibited greater mRNA accumulation than transformants 


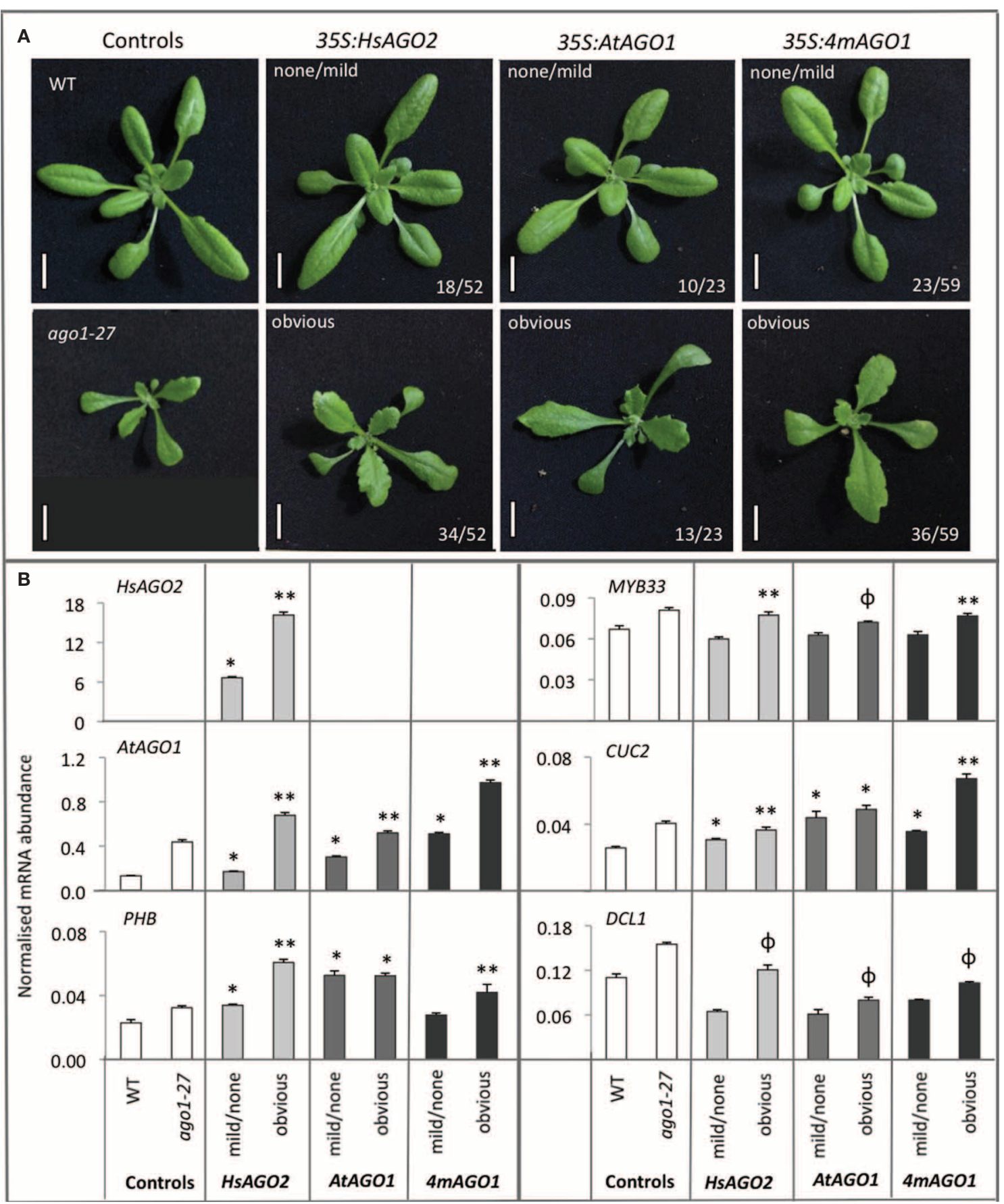

FIGURE 2 | Overexpression of HsAG02 or AtAG01 results in similar morphological and molecular phenotypes. (A) 24-day old primary transformants for the 35S:HsAGO2, 35S:AtAGO1, and 35S:4mAGO1 constructs, grown in parallel, were categorized as exhibiting an obvious abnormal morphological phenotype, characterized by broad, flattened, serrated leaves, or no/mild abnormal phenotype. Wild type (WT) and ago1-27 plants were grown in parallel as comparators. Scale bars represent $10 \mathrm{~mm}$. (B) The abundances of HsAGO2 mRNA and un-cleaved mRNA for AtAGO1, PHB, MYB33, CUC2, and DCL1 were measured in total RNA from sample pools composed of 4-8 transformants, 24-days old, from each morphological category for each construct. Wild type (WT) and ago 1-27 plants, grown in parallel, were included as controls. Measurements of "un-cleaved" mRNA are obtained by using a qRT-PCR amplicon spanning the cleavage site for each transcript, such that cleaved mRNA does not contribute to the recorded abundance. All measurements are normalized to CYCLOPHILIN mRNA. Data is averaged from two technical cDNA replicates, each of which comprised triplicate measurements, and error bars depict standard error of the mean. Values marked with * are significantly larger $(P<0.05)$ than their corresponding measurements in WT samples, those with ${ }^{*}$ are significantly larger $(P<0.05)$ than both WT and their corresponding none/mild sample, whilst those marked with $\phi$ are significantly larger $(P<0.05)$ than their corresponding none/mild sample but not WT. 
showing no/mild aberrant phenotypes or wild type comparators for AtAGO1, PHB, MYB33, and CUC2 $(P<0.05)$ (Figure 2B). The same was generally true for 35S:AtAGO1 and 35S:4mAGO1 transformants and it could not be said that, across the board, any one of the three constructs generated a more severe molecular phenotype than the other two (Figure 2B). For each construct, DCL1 levels were not significantly increased relative to wild type but were higher in transformants that displayed an obvious aberrant phenotype than those that did not $(P<0.05)$ (Figure 2B). Thus, the exhibition of morphological defects among transformants of all three constructs corresponded with a general increase in the mRNA abundance of miRNA targets, implying a perturbation of miRNA activity in each.

The apparent perturbation of miRNA activity in plants expressing $\mathrm{HsAGO} 2$ at high levels seems to imply an interaction between the HsAGO2 protein and some component/s of the endogenous miRNA pathway, the most obvious candidate being miRNA molecules themselves. Hence, mature miRNA levels for two highly abundant miRNAs, miR159a and miR166, were measured in 35S:HsAGO2 transformants from the same morphological categories as above, with 35S:AtAGO1 and 35S:4mAGO1 transformants, wild type and ago1-27 again included for comparison. Transformants of all three constructs displaying no aberrant phenotype showed miRNA accumulation roughly equivalent to wild type (Figure 3). MiR159a and miR166 levels were decreased in transformants displaying an obvious aberrant phenotype, though these decreases were not always found to be statistically significant compared to wild type $(P<0.05)$ (Figure 3 ). Since miRNA levels were also lower in ago1-27 than in wild type (Figure 3), this result is consistent with the notion that the three constructs behave as miRNA-pathway loss-of-function alleles. The decreased accumulation of mature miRNAs could not be explained by changes at the transcriptional level, since parallel decreases in pri-miRNA abundances were not observed (data not included).

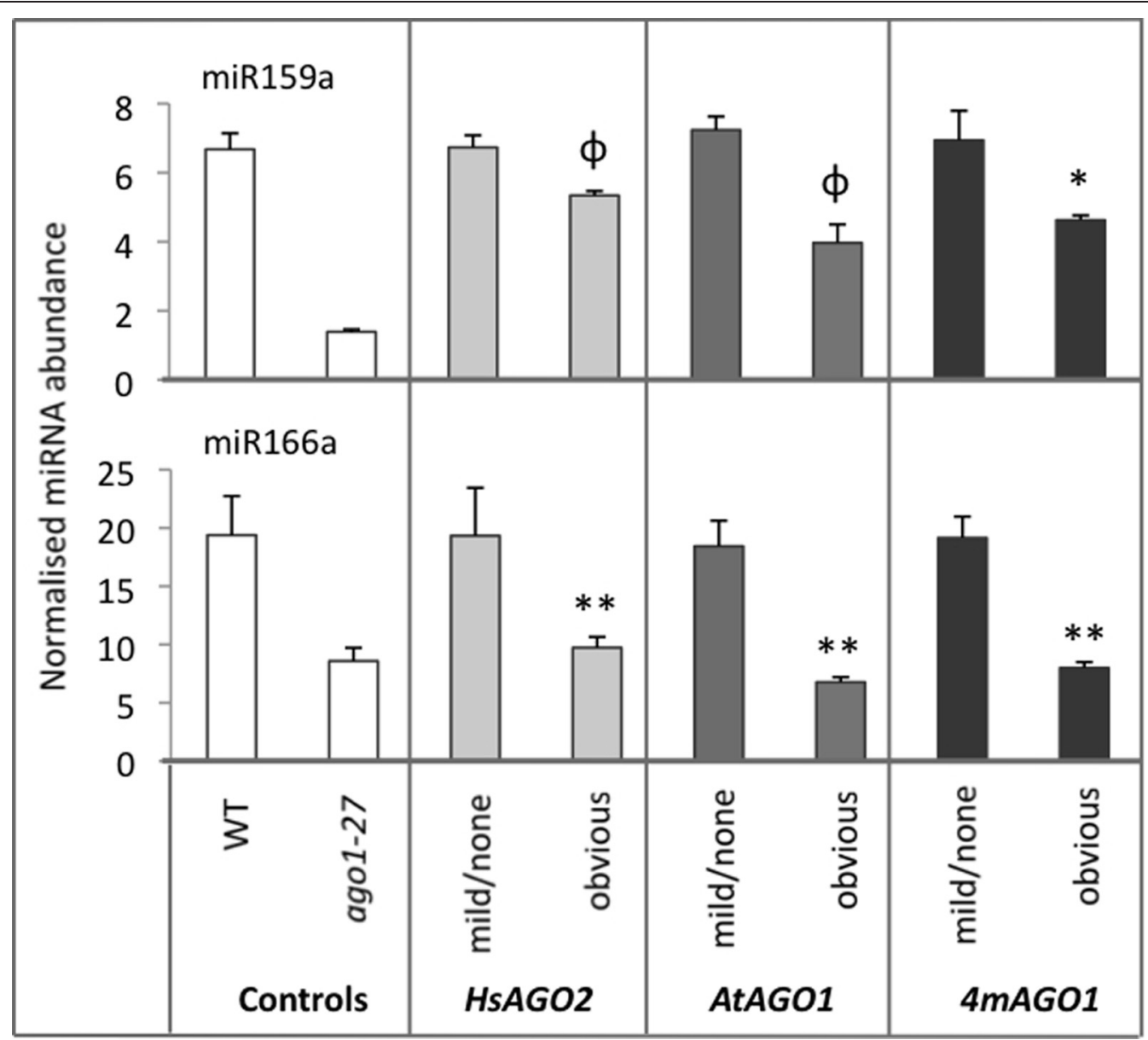

FIGURE 3 | miRNA abundances decrease in AGO overexpressing plants. The abundances of miR159a and miR166 were measured in total RNA from sample pools composed of 4-8 transformants, 24-days old, from each morphological category for each construct. Wild type (WT) and ago1-27 plants, grown in parallel, were included as controls. miRNA levels are normalized to the small RNA sno101. Data is averaged from two technical
cDNA replicates, each of which comprised triplicate measurements, and error bars depict standard error of the mean. Values marked with * are significantly smaller $(P<0.05)$ than corresponding measurement in WT samples, those with ${ }^{* *}$ are significantly smaller $(P<0.05)$ than both WT and their corresponding none/mild sample, whilst those marked with $\phi$ are significantly smaller $(P<0.05)$ than their corresponding none/mild sample but not WT. 
In sum, 35S:HsAGO2 transformants and AtAGO1 overexpressers are morphologically indistinguishable, display similarly elevated mRNA abundances for five miRNA targets and comparable reductions in the accumulation of two mature miRNAs. Collectively, these findings strongly argue that HsAGO2 inhibits endogenous miRNA activity and behaves in a similar fashion to its plant counterpart, at least in an over-expression context.

\section{S:HsAG02 IS UNABLE TO RESCUE THE ago1-27 ALLELE}

That HsAGO2 expression generates a miRNA-pathway loss-offunction phenotype does not necessarily imply that the protein lacks functionality in the plant cell, since the over-expression of AtAGO1 similarly inhibits endogenous miRNA activity. With the anticipation that a functional $\mathrm{HsAGO} 2$ protein might alleviate an agol loss-of-function allele, the 35S:HsAGO2 construct was transformed into ago1-27, as were 35S:AtAGO1 and 35S:4mAGO1. Both AtAGO1 constructs were able to complement the ago1-27 morphological phenotype, yielding transformants resembling wild type plants with similar frequencies. Full or partial complementation was observed in 15/38 transformants of 35S:AtAGO1 and 20/46 for 35S:4mAGO1 (Figure 4). This implies that, although both constructs can inhibit miRNA activity in a wild type background, they are also able to fully or partially restore AGO1 activity in the ago1-27 mutant background. By contrast, none of the 68 transformants for 35S:HsAGO2 showed even partial phenotypic complementation, with the majority exhibiting an even more severely aberrant phenotype than ago1-27 (Figure 4). This result argues that, whilst HsAGO2 behaves just like AtAGO1 in an over-expression context, the human protein, unlike its plant counterpart, is seemingly incapable of facilitating the efficient silencing of miRNA targets in Arabidopsis.

\section{DISCUSSION}

In both plants and animals, an AGO protein is required for miRNA-mediated gene silencing. The best-studied AGO proteins of each kingdom, AtAGO1 and HsAGO2 are believed to share a high degree of functional conservation. Seeking to determine the full extent of their similarity, we constitutively expressed HsAGO2 in Arabidopsis. 35S:HsAGO2 transformants displayed abnormal phenotypes indistinguishable from those of transgenic plants over-expressing AtAGO1, with both groups resembling miRNA-pathway loss-of-function alleles at the morphological and molecular levels and exhibiting similar decreases in the abundances of mature miRNAs. We are aware of no other attempt to express a component of the animal miRNA pathway in planta, or vice versa, and our results indicate that, despite the evolutionary gulf between the two systems, the HsAGO2 protein retains the ability to interact with some component/s of the plant miRNA pathway and behaves similarly to AtAGO1 in an over-expression context.

Whilst HsAGO2 behaves just like AtAGO1 in an overexpression context, it was unable to rescue the ago1-27 allele, indicating that it is insufficient for the efficient silencing of miRNA targets in Arabidopsis. This is unsurprising, given that

\begin{tabular}{|c|c|c|c|}
\cline { 2 - 4 } \multicolumn{1}{c|}{} & \multicolumn{3}{c|}{ Complementation frequency } \\
\cline { 2 - 4 } \multicolumn{1}{c|}{} & None & Partial & Full \\
\hline 35 S:HsAGO2 & 68 & 0 & 0 \\
\hline 35 S:AtAGO1 & 23 & 12 & 3 \\
\hline $355: 4 m A G O 1$ & 26 & 13 & 7 \\
\hline
\end{tabular}

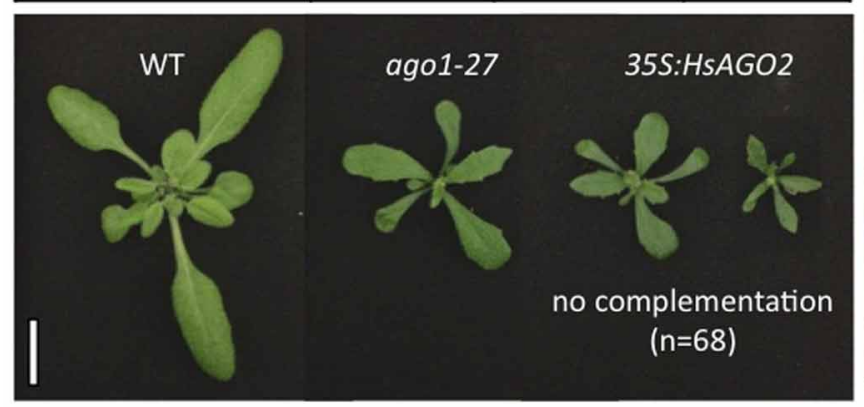

FIGURE 4 | HsAGO2 cannot complement the ago1-27 mutation. 22-day old transformants of 35S:HsAGO2, 35S:AtAGO1, and 35S:4mAGO1 in the ago 1-27 background were categorized based on their morphologies as being non-complemented (resembling ago1-27), partially complemented (intermediate phenotype) or fully complemented (resembling wild type). The

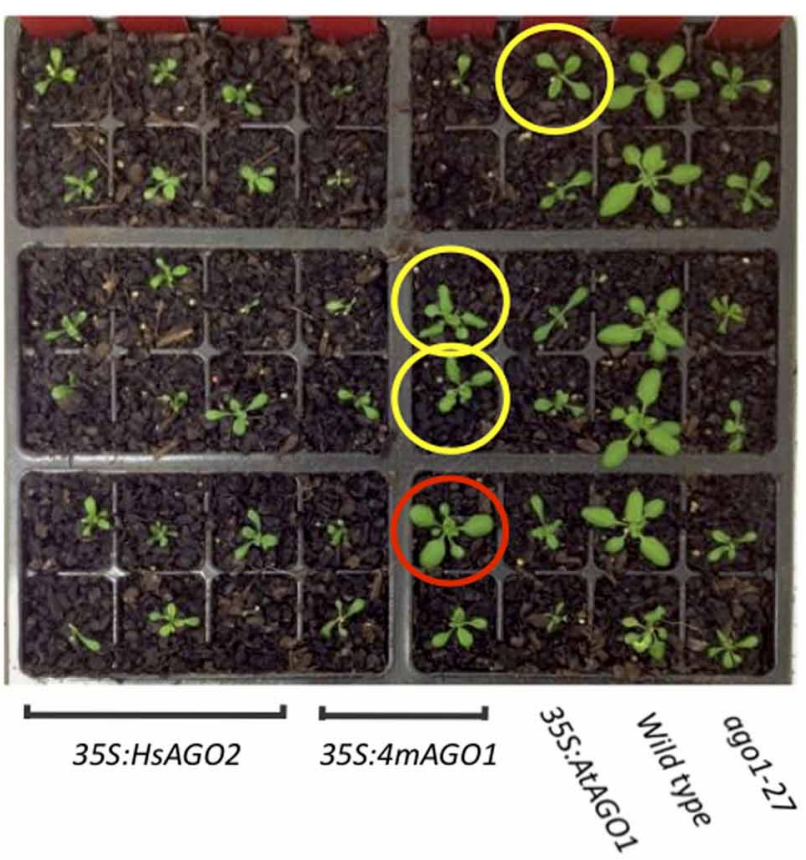

left image shows representative non-complemented transformants for the 35S:HsAGO2 construct, alongside wild type (WT) and ago1-27 controls. Scale bar represents $10 \mathrm{~mm}$. The right image shows a representative group of transformants for each construct, alongside WT and ago 1-27, with plants that were scored as being partially (yellow) or fully complemented (red) highlighted. 
even the closest homologue of AtAGO1, AtAGO10 — with which it shares $75 \%$ amino acid identity compared to just $43 \%$ for HsAGO2 (Poulsen et al., 2013) —expressed from AtAGO1's promoter, is unable to rescue the same allele (Zhu et al., 2011). In fact, ectopic expression of AtAGO10 from the AtAGO1 promoter yielded upward leaf-curl and exacerbated the ago1-27 allele (Zhu et al., 2011), just as we observed for the 35S:HsAGO2 construct. While AtAGO10 associates with miRNAs and is capable of cleaving target transcripts in vitro, it is not an efficient gene-silencer in vivo and instead attenuates AtAGO1-associated miRNA activity by sequestering miRNAs, having a particular preference for miR165/166 (Zhu et al., 2011). The HsAGO2 protein is also sufficient for sRNA-directed mRNA cleavage in vitro (Liu et al., 2004; Meister et al., 2004; Rivas et al., 2005) but apparently fails to efficiently silence miRNA targets in planta. It seems therefore that a basal cleavage activity, demonstrable in vitro, is not sufficient for proper miRNA-mediated gene silencing in planta. Consequently, we suggest that the AtAGO1 protein is distinguished by some specialization, perhaps allowing it to interact with an unknown factor/s that is additionally required for efficient silencing, which is essential for its function. The requirement for an additional factor/s could explain the somewhat counterintuitive observation that AtAGO1 over-expression generates a miRNA-pathway loss-of-function effect; an excess of AtAGO1 protein might titrate miRNAs and other necessary components of the miRISC into separate, incomplete complexes, each unable to facilitate miRNAguided gene silencing, thereby inhibiting endogenous miRNA activity. Such an explanation was proposed by Vaucheret et al. (2004), who first reported a perturbation of miRNA activity by AtAGO1 over-expression. Here it must be noted that Vaucheret et al. (2006) reported modest increases in the accumulation of mature miR159a and miR166 in transgenic plants over-expressing AtAGO1, the opposite result to what we observed. The foundation of this contradiction might lie in the fact that the two studies employed different protocols for the extraction of total RNA from plant tissues, although this explanation cannot be substantiated here. That both the AtAGO1 and the $4 m A G O 1$ transgenes were,

\section{REFERENCES}

Axtell, M. J., Westholm, J. O., and Lai, E. C. (2011). Vive la difference: biogenesis and evolution of microRNAs in plants and animals. Genome Biol. 12:221. doi: $10.1186 / \mathrm{gb}-2011-12-4-$ 221

Baumberger, N., and Baulcombe, D. C. (2005). Arabidopsis ARGONAUTE1 is an RNA Slicer that selectively recruits microRNAs and short interfering RNAs. Proc. Natl. Acad. Sci. U.S.A. 102, 11928-11933.

Brodersen, P., Sakvarelidze-Achard, L., Bruun-Rasmussen, M., Dunoyer, P., Yamamoto, Y. Y., Sieburth, L., et al. (2008). Widespread translational inhibition by plant miRNAs and siRNAs. Science 320, 1185-1190.

Brodersen, P., and Voinnet, O. (2009). Revisiting the principles of

in the present study, expressed from a constitutive double $35 \mathrm{~S}$ promoter in, as opposed to the endogenous promoter of AtAGO1 (Vaucheret et al., 2006), is another point of difference between the two approaches, and may have contributed to their divergent outcomes.

The miRNA pathways of plants and animals utilize similar molecular machinery but exhibit characteristic distinctions in the manner of their operation, presumably the manifestation of componential modifications that have accumulated throughout their parallel evolutionary histories. The most important of these is the differential requirements for target recognition in each, animal miRNAs commonly regulating targets that would be ignored in plants for their insufficient complementarity (Axtell et al., 2011). Given the intimacy of their involvement in miRNAmediated gene silencing, one could reasonably speculate that some intrinsic difference in the AGO proteins of plants and animals might underpin their differential target specificities. Because HsAGO2 was apparently unable to efficiently silence miRNA targets in planta, we were unable to address the possibility that an animal and a plant AGO might regulate distinct sets of targets when expressed in an identical cellular context. However, it was recently reported that cleavage-impaired AtAGO1 mutants, which are defective in gene silencing activity, successfully coprecipitate with both miRNAs and mRNAs, suggesting that their ability to recognize and bind to target transcripts remains intact (Carbonell et al., 2012). Thus, an avenue for future work will be to attempt to elucidate the target profile of HsAGO2 in planta via co-precipitation experiments, potentially revealing differential specificities for an animal and plant AGO.

\section{ACKNOWLEDGMENTS}

This work was funded by the Australian Research Council Discovery grant DP11013493. We would like to thank Dr. Hervé Vaucheret for the ago1-27 seeds. We thank the Arabidopsis Biological Resource Center for providing the AtAGO1 clone. We thank Rob Allen and Marlene Reichel for comments to the manuscript.

40 ortholog SQUINT to Hsp90 protein is required for SQUINT function in Arabidopsis. J. Biol. Chem. 286, 38184-38189.

Eulalio, A., Rehwinkel, J., Stricker, M., Huntzinger, E., Yang, S. F., Doerks, T., et al. (2007). Target-specific requirements for enhancers of decapping in miRNA-mediated gene silencing. Genes Dev. 21, 2558-2570.

Frank, F., Sonenberg, N., and Nagar, B. (2010). Structural basis for $5^{\prime}$ nucleotide base-specific recognition of guide RNA by human AGO2 Nature 465, 818-822.

Huntzinger, E., and Izaurralde, E. (2011). Gene silencing by microRNAs: contributions of translational repression and mRNA decay. Nat. Rev. Genet. 12, 99-110.
Iki, T., Yoshikawa, M., Meshi, T., and Ishikawa, M. (2012). Cyclophilin 40 facilitates HSP90-mediated RISC assembly in plants. EMBO J. 31, 267-278.

Iki, T., Yoshikawa, M., Nishikiori, M., Jaudal, M. C., MatsumotoYokoyama, E., Mitsuhara, I., et al. (2010). In vitro assembly of plant RNA-induced silencing complexes facilitated by molecular chaperone HSP90. Mol. Cell 39, 282-291.

Iwasaki, S., Kobayashi, M., Yoda, M., Sakaguchi, Y., Katsuma, S., Suzuki, T., et al. (2010). Hsc70/Hsp90 chaperone machinery mediates ATP-dependent RISC loading of small RNA duplexes. Mol. Cell 39, 292-299.

Lanet, E., Delannoy, E., Sormani, R., Floris, M., Brodersen, P., Crete, P., 
et al. (2009). Biochemical evidence for translational repression by Arabidopsis microRNAs. Plant Cell 21, 1762-1768.

Liu, H., and Naismith, J. H. (2008). An efficient one-step site-directed deletion, insertion, single and multiplesite plasmid mutagenesis protocol. BMC Biotechnol. 8:91. doi: 10.1186/1472-6750-8-91

Liu, J., Carmell, M. A., Rivas, F. V., Marsden, C. G., Thomson, J. M., Song, J. J., et al. (2004). Argonaute2 is the catalytic engine of mammalian RNAi. Science 305, 1437-1441.

Mallory, A., and Vaucheret, H. (2010). Form, function, and regulation of ARGONAUTE proteins. Plant Cell 22, 3879-3889.

Meister, G., Landthaler, M., Patkaniowska, A., Dorsett, Y., Teng, G., and Tuschl, T. (2004). Human Argonaute2 mediates RNA cleavage targeted by miRNAs and siRNAs. Mol. Cell 15, 185-197.

Mi, S., Cai, T., Hu, Y., Chen, Y., Hodges, E., Ni, F., et al. (2008). Sorting of small RNAs into Arabidopsis argonaute complexes is directed by the $5^{\prime}$ terminal nucleotide. Cell 133, 116-127.

Morel, J. B., Godon, C., Mourrain, P., Beclin, C., Boutet, S., Feuerbach, F., et al. (2002). Fertile hypomorphic ARGONAUTE (agol) mutants impaired in post-transcriptional gene silencing and virus resistance. Plant Cell 14, 629-639.
Nakanishi, K., Weinberg, D. E., Bartel, D. P., and Patel, D. J. (2012). Structure of yeast Argonaute with guide RNA. Nature 486, 368-374.

Pasquinelli, A. E. (2012). MicroRNAs and their targets: recognition, regulation and an emerging reciprocal relationship. Nat. Rev. Genet. 13, 271-282.

Pillai, R. S., Artus, C. G., and Filipowicz, W. (2004). Tethering of human Ago proteins to mRNA mimics the miRNA-mediated repression of protein synthesis. RNA 10, 1518-1525.

Poulsen, C., Vaucheret, H., and Brodersen, P. (2013). Lessons on RNA silencing mechanisms in plants from eukaryotic argonaute structures. Plant Cell 25, 22-37.

Rivas, F. V., Tolia, N. H., Song, J. J., Aragon, J. P., Liu, J., Hannon, G. J., et al. (2005). Purified Argonaute2 and an siRNA form recombinant human RISC. Nat. Struct. Mol. Biol. 12, 340-349.

Schirle, N. T., and Macrae, I. J. (2012). The crystal structure of human Argonaute2. Science 336, 1037-1040.

Smith, M. R., Willmann, M. R., Wu, G., Berardini, T. Z., Moller, B., Weijers, D., et al. (2009). Cyclophilin 40 is required for microRNA activity in Arabidopsis. Proc. Natl. Acad. Sci. U.S.A. 106, 5424-5429.

Takimoto, K., Wakiyama, M., and Yokoyama, S. (2009). Mammalian GW182 contains multiple
Argonaute-binding sites and functions in microRNA-mediated translational repression. RNA 15 , 1078-1089.

Vaucheret, H. (2008). Plant ARGONAUTES. Trends Plant Sci. 13, 350-358.

Vaucheret, H., Mallory, A. C., and Bartel, D. P. (2006). AGO1 homeostasis entails coexpression of MIR168 and AGO1 and preferential stabilization of miR168 by AGO1. Mol. Cell 22, 129-136.

Vaucheret, H., Vazquez, F., Crete, P. and Bartel, D. P. (2004). The action of ARGONAUTE1 in the miRNA pathway and its regulation by the miRNA pathway are crucial for plant development. Genes Dev. 18, 1187-1197.

Vazquez, F., Gasciolli, V., Crete, P., and Vaucheret, H. (2004). The nuclear dsRNA binding protein HYL1 is required for microRNA accumulation and plant development, but not posttranscriptional transgene silencing. Curr. Biol. 14, 346-351.

Yang, L., Wu, G., and Poethig, R S. (2012). Mutations in the GWrepeat protein SUO reveal a developmental function for microRNAmediated translational repression in Arabidopsis. Proc. Natl. Acad. Sci. U.S.A. 109, 315-320.

Zhu, H., Hu, F., Wang, R., Zhou, X., Sze, S. H., Liou, L. W., et al. (2011). Arabidopsis Argonaute10 specifically sequesters miR166/165 to regulate shoot apical meristem development. Cell 145, 242-256.

Zipprich, J. T., Bhattacharyya, S., Mathys, H., and Filipowicz, W. (2009). Importance of the Cterminal domain of the human GW182 protein TNRC6C for translational repression. RNA 15, 781-793.

Conflict of Interest Statement: The authors declare that the research was conducted in the absence of any commercial or financial relationships that could be construed as a potential conflict of interest.

Received: 13 February 2013; accepted: 28 March 2013; published online: 16 April 2013

Citation: Deveson I, Li J and Millar $A A$ (2013) Expression of human ARGONAUTE 2 inhibits endogenous microRNA activity in Arabidopsis. Front. Plant Sci. 4:96. doi: 10.3389/fpls. 2013.00096

This article was submitted to Frontiers in Plant Genetics and Genomics, a specialty of Frontiers in Plant Science.

Copyright (c) 2013 Deveson, Li and Millar. This is an open-access article distributed under the terms of the Creative Commons Attribution License, which permits use, distribution and reproduction in other forums, provided the original authors and source are credited and subject to any copyright notices concerning any third-party graphics etc. 